\title{
Viable rat offspring derived from oocytes intracytoplasmically injected with freeze-dried sperm heads
}

\author{
Masumi Hirabayashi ${ }^{1,2 *}$, Megumi Kato ${ }^{1 *}$, Junya Ito ${ }^{1}$ and Shinichi Hochi ${ }^{3}$ \\ National Institute for Physiological Sciences, The Graduate University for Advanced Studies, and Shinshu University, Japan
}

Date submitted: 08.10.04. Date accepted: 25.11.04

\section{Summary}

\begin{abstract}
A $2 \times 3$ factorial designed experiment was conducted in order to examine whether freeze-dried rat spermatozoa can participate in full-term development following intracytoplasmic sperm injection (ICSI). A sperm suspension from cauda epididymides of Sprague-Dawley (SD) rats was prepared with or without ultrasonic treatment. The sonicated and non-sonicated sperm suspensions were processed for freeze-thawing (FT groups; $100 \mu \mathrm{l}$ sample was cooled in liquid nitrogen vapor, stored for 1 day at $196^{\circ} \mathrm{C}$, and thawed in a $25^{\circ} \mathrm{C}$ water bath) and freeze-drying (FD groups; $100 \mu \mathrm{l}$ sample was frozen in liquid nitrogen for $20 \mathrm{~s}$, lyophilized for $6 \mathrm{~h}$, stored at $4{ }^{\circ} \mathrm{C}$ for 2 days, and rehydrated with $100 \mu \mathrm{l}$ ultrapure water), or were subjected to immediate use for ICSI (fresh control groups). The sperm heads were microinjected into denuded SD oocytes using a piezo-driven micropipette $2-4 \mu \mathrm{m}$ in diameter. The presumptive zygotes were transferred into oviducts of pseudopregnant Wistar female rats. Viable rat offspring were produced from all six experimental groups. Ultrasonic treatment of rat spermatozoa was effective in increasing the offspring rate $(23.3 \%$ vs $6.7 \%$ in fresh control groups, $35.0 \%$ vs $7.6 \%$ in FT groups, $9.2 \%$ vs $2.5 \%$ in FD groups). The acrosomal region appeared to be intact even after ultrasonic FT and FD treatments as well as in the fresh controls, while the lateral dorsal region of the sperm membrane was more or less damaged in the sonicated, FT and FD samples. Thus, the successful participation of freeze-dried spermatozoa in full-term development was demonstrated by applying ICSI in the rat.
\end{abstract}

Keywords: Freeze-dry, ICSI, Rat oocytes, Sonication

\section{Introduction}

Since freeze-dried spermatozoa can be stored at an ambient or refrigerated temperature, the costs required for the maintenance and shipping of spermatozoa would be reduced. To date, viable offspring in mice (Wakayama \& Yanagimachi, 1998; Kusakabe et al., 2001; Kaneko et al., 2003a, b; Ward et al., 2003) and rabbits (Liu et al., 2004) have been produced by intracytoplasmic sperm injection (ICSI) using freeze-dried samples.

All correspondence to: Dr M. Hirabayashi, Center for Brain Experiment, National Institute for Physiological Sciences, Okazaki, Aichi 444-8787, Japan. Tel: +81 56459 5265. Fax: +81564595266. e-mail: mhirarin@nips.ac.jp

* M.H. and M.K. contributed equally to this work.

${ }^{1}$ National Institute for Physiological Sciences, Okazaki, Aichi 444-8787, Japan.

${ }^{2}$ School of Life Science, The Graduate University for Advanced Studies, Okazaki, Aichi 444-8787, Japan.

${ }^{3}$ Faculty of Textile Science and Technology, Shinshu University, Ueda, Nagano 386-8567, Japan.
Blastocyst development after ICSI with freeze-dried sperm heads was also reported in large domestic animals including cattle (Keskintepe et al., 2002) and pigs (Kwon et al., 2004).

Spermatozoal centrosomes in rodents do not contribute to zygotic cleavage (Schatten et al., 1991), while those in other mammalian species (e.g. rabbit, pig, cattle and human) serve as a microtubuleorganizing centre in the zygotes (Yllera-Fernandez et al., 1992; Palermo et al., 1997). Therefore, in rodents, treatments to dissociate the long sperm tail/mid-piece from the spermatozoon, such as freeze-thawing or sonication (Kuretake et al., 1996; Hirabayashi et al., 2005), would facilitate the handling and microinjection of spermatozoa. The ultrasonic treatment of mouse spermatozoa had a negative impact (Kuretake et al., 1996) or a negligible influence (Tateno et al., 2000) on the developmental competence of ICSI oocytes. On the other hand, mouse, hamster and human spermatozoa microinjected into hamster oocytes easily underwent decondensation of the sperm heads, but 
the decondensation of rat and bovine spermatozoa was quite time-consuming (Perreault et al., 1988).

As for rats, we have recently established the protocols for microinsemination, such as ICSI (Hirabayashi et al., 2002a; Kato et al., 2004a), elongating spermatid injection (ELSI; Kato et al., 2004a), or round spermatid injection (ROSI; Hirabayashi et al., 2002b; Kato et al., 2004b). The offspring rate in rat ICSI (Kato et al., 2004a) approaches the level achieved in practice by mouse ICSI (Ogura et al., 2003). The objectives of the present study were to investigate whether freeze-dried rat spermatozoa are capable of participating in full-term development by ICSI, and whether the ultrasonic treatment of rat spermatozoa affects the production efficiency of the ICSI-derived offspring.

\section{Materials and methods}

\section{Media}

The medium used for the suspension, sonication, freeze-thawing and freeze-drying of the epididymal spermatozoa was $10 \mathrm{mM}$ Tris-buffer (Nacalai Tesque, Kyoto, Japan) supplemented with $50 \mathrm{mM} \mathrm{NaCl}$ (Wako Pure Chemical Industries, Osaka, Japan) and $50 \mathrm{mM}$ EGTA (Sigma-Aldrich, St Louis, MO), referred to hereafter as Tris/EGTA (Kusakabe et al., 2001). The medium used for the collection and temporal incubation of the oocytes and for the culture and transfer of the ICSI oocytes was modified rat 1-cell embryo culture medium, mR1ECM (Oh et al., 1998), containing $110 \mathrm{mM}$ $\mathrm{NaCl}$ and $0.4 \%$ fatty-acid-free bovine serum albumin (BSA) (Sigma-Aldrich), referred to hereafter as $\mathrm{mR} 1 \mathrm{ECM} / \mathrm{BSA}$. The mR1ECM/BSA was modified by the supplementation of Hepes (Wako) at $22 \mathrm{mM}$ and the reduction of sodium bicarbonate to $5 \mathrm{mM}$, referred to hereafter as Hepes-R1ECM, and used for ICSI. HepesR1ECM supplemented with $12 \%$ polyvinylpyrrolidone (PVP, 360 kDa; ICN Pharmaceuticals, Costa Mesa, CA) was used for placing the sperm heads immediately before their injection.

\section{Animals}

Specific pathogen-free Slc:SD 4- to 5-week-old female rats and Slc:SD 10-week-old male rats (Japan SLC, Shizuoka, Japan) were used as oocyte donors and sperm donors, respectively. Crj:Wistar female rats that were 8-13 weeks old (Charles River Japan, Kanagawa, Japan) were used as the surrogate mothers. The rats were housed in an environmentally controlled room with a $12 \mathrm{~h}$ dark to $12 \mathrm{~h}$ light cycle at a temperature of $23 \pm 2{ }^{\circ} \mathrm{C}$ and humidity of $55 \pm 5 \%$, and given free access to a laboratory diet (MF; Oriental Yeast, Tokyo, Japan) and filtered water.

\section{Sperm preparation}

Spermatozoa from a pair of cauda epididymides were allowed to disperse in $2 \mathrm{ml}$ of Tris/EGTA for $30 \mathrm{~min}$ and then collected in a $5 \mathrm{ml}$ tube. The swim-up sperm in the upper $1 \mathrm{ml}$ were divided into two groups. The first $0.5 \mathrm{ml}$ of the sperm suspension was sonicated for 10 s using a $10 \%$ power output from an ultrasonic cell disrupter (Sonifier 250; Branson, Danbury, CT), while the remaining $0.5 \mathrm{ml}$ was kept as the non-sonicated sample. Both the sonicated and non-sonicated sperm samples were allocated to one of three groups: freezethawed, freeze-dried or fresh control.

In each of the freeze-thawed groups, $100 \mu \mathrm{l}$ of the sperm suspension was transferred to a $1.0 \mathrm{ml}$ cryotube (Nunc, Roskilde, Denmark), frozen in liquid nitrogen vapor for $1 \mathrm{~min}$, and stored at $-196^{\circ} \mathrm{C}$ for $24 \mathrm{~h}$. The sample was thawed in a $25^{\circ} \mathrm{C}$ water bath for $1 \mathrm{~min}$.

In each of the freeze-dried groups, $100 \mu l$ of the sperm suspension was transferred to a $1.5 \mathrm{ml}$ polypropylene tube (Eppendorf, Hamburg, Germany), frozen in liquid nitrogen for $20 \mathrm{~s}$, and lyophilized for $6 \mathrm{~h}$ by a connected freeze-drying apparatus (Yamato Scientific, Tokyo, Japan). After lyophilization, the tubes were sealed with parafilm under air and stored at $4{ }^{\circ} \mathrm{C}$ for 2 days. The sample was rehydrated by adding $100 \mu \mathrm{l}$ of ultrapure water (Sigma-Aldrich) to the tube.

\section{Morphological evaluation of sperm membrane}

The membrane and acrosomal status of the rat spermatozoa after sonication, freeze-thawing and freezedrying was compared with that of the non-treated fresh spermatozoa, as previously described (Fazeli et al., 1997). Briefly, a smeared sperm suspension $(3 \mu \mathrm{l})$ on a microscope slide was air-dried and fixed with absolute methanol for $10 \mathrm{~min}$. The smear was counterstained with a total of $5 \mu \mathrm{l} \mathrm{of} 5 \mu \mathrm{g} / \mathrm{ml}$ fluorescein isothiocyanate (FITC)-labeled peanut agglutinin (PNA, Arachis hypogaea) (FITC-PNA; Sigma-Aldrich) and $4 \mu \mathrm{M}$ ethidium homodimer-1 (EthD-1; Molecular Probes, Eugene, OR) for $30 \mathrm{~min}$ at $37^{\circ} \mathrm{C}$. After being rinsed with phosphate-buffered saline (PBS) and mounted with coverslips, the slides were examined under an epifluorescence microscope (TE-300; Nikon, Tokyo, Japan) equipped with a DFT set of filters. Approximately 40-50 spermatozoa per treatment group were observed.

\section{ICSI}

The ICSI experiments were performed for three successive days (fresh groups on day 1, freeze-thawed groups on day 2, freeze-dried groups on day 3 ) and replicated three times. Female rats were superovulated by intraperitoneal injections of $300 \mathrm{IU} / \mathrm{kg}$ equine chorionic gonadotropin (eCG; Nippon Zenyaku 
Kogyo, Fukushima, Japan) and $300 \mathrm{IU} / \mathrm{kg}$ human chorionic gonadotropin (hCG; Sankyo Yell Yakuhin, Tokyo, Japan) at an interval of $48 \mathrm{~h}$ (Hirabayashi et al., 2001). The cumulus-oocyte complexes were collected from the oviductal ampullae 14-17 h after the hCG injection and were freed from the cumulus cells by a $5 \mathrm{~min}$ treatment with $0.1 \%$ hyaluronidase (SigmaAldrich). The denuded oocytes were washed three times with mR1ECM/BSA and incubated for up to $45 \mathrm{~min}$ at $37^{\circ} \mathrm{C}$ in $5 \% \mathrm{CO}_{2}$ in air.

The sperm heads were injected into the denuded oocytes at ambient temperature $\left(23 \pm 2{ }^{\circ} \mathrm{C}\right)$ using a piezo impact-driving unit (PMM-140FU; Prime Tech, Ibaraki, Japan) with a pulse controller (PMAS-CT140; Prime Tech), as previously described (Hirabayashi et al., 2002a). Briefly, the sperm head that hung on the blunt end of the $2-4 \mu \mathrm{m}$ diameter injection pipette was released near the oocyte. After zona drilling by a few piezo pulses, the sperm head was hung on the blunt end of the pipette again. The pipette tip was mechanically advanced deep into the oocyte, significantly stretching the oolemma. Upon application of a light positive pressure and a piezo pulse, the oolemma was punctured at the pipette tip and the sperm head was ejected into the oocyte. The injection pipette was then quickly withdrawn.

\section{Culture and transfer of ICSI oocytes}

The ICSI oocytes were washed three times with mR1ECM/BSA and cultured in $100 \mu \mathrm{l}$ microdrops of the same medium covered with mineral oil (SigmaAldrich) at $37^{\circ} \mathrm{C}$ in $5 \% \mathrm{CO}_{2}$ in air. The morphological survival rates and cleavage rates were assessed at 6 and $24 \mathrm{~h}$, respectively, after completing the ICSI. Oocyte activation rate was also assessed at $6 \mathrm{~h}$ after the ICSI. Presumptive zygotes at the 2-cell stage and nondegenerating 1-cell stage were harvested at $24 \mathrm{~h}$ postICSI, and then transferred into the oviductal ampullae of the recipient rats which had been previously mated with a vasectomized male rat. Embryo transfer to the pseudopregnant recipients (12-34 embryos per recipient) was performed on the day that the vaginal plug was detected. The pregnant recipients were allowed to deliver the offspring.

\section{Statistical analysis}

The percentage data in each replicate were arcsintransformed before being subjected to one-way analysis of variance. Significant differences were determined by Fisher's protected least significant difference test using the StatView program (Abacus Concepts, Berkeley, CA). A value of $p<0.05$ was chosen as an indication of statistical significance.

\section{Results}

The ultrasonic treatment of the rat spermatozoa completely separated their tails / mid-pieces from the sperm heads, while less than $10 \%$ of the spermatozoa after freeze-thawing or freeze-drying were free of their tails /mid-pieces. Almost all spermatozoa in each treatment showed similar morphological characteristics in their acrosome and plasma membrane. As shown in Fig. 1, the staining of the sperm heads with FITCPNA and EthD-1 demonstrated more damage to the sperm membrane in the sonicated, freeze-thawed and freeze-dried sperm samples than in the nontreated sperm samples. The sperm membrane in the lateral dorsal region of the sonicated sample (Fig. 1b) and the freeze-dried sample (Fig. 1d) appeared to be more disrupted than that in the freeze-thawed sample (Fig. 1c). Meanwhile, the acrosomal region (tip of sperm head) appeared to be intact irrespective of the type of sample (Fig. 1a-d).

The in vitro development of rat oocytes microinseminated with fresh, freeze-thawed and freeze-dried spermatozoa with or without ultrasonic treatment is shown in Table 1. There were no significant differences in the proportions of oocytes that survived ICSI among the six experimental groups (83.6-91.4\%). Oocyte activation rates were also comparable among the groups (70.2$92.9 \%$ ) except for non-sonicated/freeze-thawed group (33.6\%). However, the oocytes microinseminated with sonicated sperm heads showed higher initial cleavage rates, especially in the fresh and freeze-thawed groups ( $35.4 \%$ and $48.6 \%$, respectively).

The full-term developmental potential of the microinseminated rat oocytes is shown in Table 2 . The oocytes microinseminated with sonicated sperm heads showed higher offspring rates in the fresh and freeze-thawed groups ( $23.3 \%$ and $35.0 \%$, respectively). The ICSI oocytes with sonicated/freeze-dried sperm heads developed into live offspring at a significantly lower rate $(9.2 \%)$ than those from the sonicated / freezethawed sperm heads $(35.0 \%, p<0.05)$. The difference in the offspring rate between the sonicated/fresh group $(23.3 \%)$ and sonicated/freeze-dried group $(9.2 \%)$ was likely to be of statistical significance $(p=0.06)$. Most of the rat offspring were born viable and appeared to be normal in size, except for one stillborn in the sonicated/freeze-dried group.

\section{Discussion}

The ICSI-derived rat offspring obtained in the present study using freeze-dried spermatozoa demonstrate that the rat is the third species after the mouse (Wakayama \& Yanagimachi, 1998) and rabbit (Liu et al., 2004) whose freeze-dried spermatozoa can 

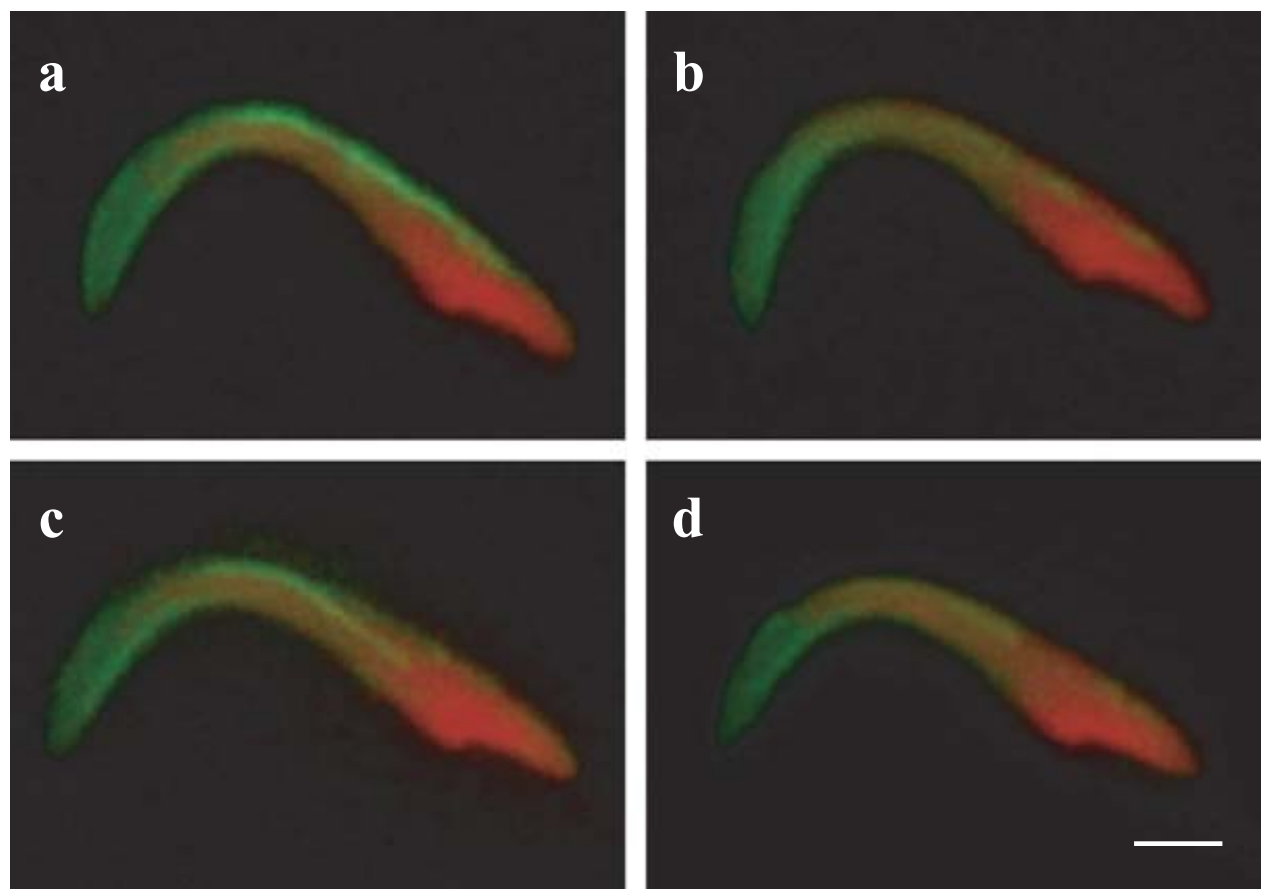

Figure 1 Acrosomal (green: FITC-PNA) and nuclear (red: EthD-1) status of the rat sperm heads. The acrosomal region (tip of sperm head) appeared to be intact irrespective of the treatments, while the lateral dorsal region of the sperm membrane was more or less damaged in the sonicated, the freeze-thawed and the freeze-dried samples. (a) Non-treated fresh control. (b) Ultrasonic treated. (c) Freeze-thawed. (d) Freeze-dried. Scale bar represents $2 \mu \mathrm{m}$.

Table 1 In vitro survival, activation and first cleavage of rat oocytes microinseminated with fresh, freeze-thawed and freeze-dried spermatozoa

\begin{tabular}{|c|c|c|c|c|c|}
\hline \multirow{2}{*}{$\begin{array}{l}\text { Sperm storage } \\
\text { condition }\end{array}$} & \multirow{2}{*}{$\begin{array}{l}\text { Ultrasonic } \\
\text { treatment }\end{array}$} & \multicolumn{4}{|c|}{ No. (\%) of oocytes } \\
\hline & & Injected & Survived & Activated & Cleaved \\
\hline \multirow[t]{2}{*}{ Fresh control } & - & 101 & $92(91.1)$ & $68(73.9)^{a}$ & $14(15.2)^{a b c}$ \\
\hline & + & 109 & $99(90.8)$ & $87(87.9)^{a}$ & $35(35.4)^{b c}$ \\
\hline \multirow[t]{2}{*}{ Freeze-thawed } & - & 146 & $122(83.6)$ & $41(33.6)^{b}$ & $9(7.4)^{a b}$ \\
\hline & + & 116 & $105(90.5)$ & $96(91.4)^{a}$ & $51(48.6)^{c}$ \\
\hline \multirow[t]{2}{*}{ Freeze-dried } & - & 136 & $124(91.2)$ & $87(70.2)^{a}$ & $0(0.0)^{a}$ \\
\hline & + & 139 & $127(91.4)$ & $118(92.9)^{a}$ & $10(7.9)^{a b}$ \\
\hline
\end{tabular}

${ }^{a-c}$ Different superscripts within columns denote significant differences at $p<0.05$.

Table 2 Full-term development of rat oocytes microinseminated with fresh, freeze-thawed and freeze-dried spermatozoa

\begin{tabular}{lcccc}
\hline $\begin{array}{l}\text { Sperm storage } \\
\text { condition }\end{array}$ & $\begin{array}{c}\text { Ultrasonic } \\
\text { treatment }\end{array}$ & $\begin{array}{c}\text { No. of oocytes transferred } \\
\text { [Range per recipient] }\end{array}$ & $\begin{array}{c}\text { No. of recipients } \\
\text { pregnant/used }\end{array}$ & $\begin{array}{c}\text { No. (\%) of } \\
\text { offspring }\end{array}$ \\
\hline Fresh control & - & $90[16-34]$ & $2 / 4$ & $6(6.7)^{a}$ \\
& + & $86[14-28]$ & $4 / 4$ & $20(23.3)^{b c}$ \\
Freeze-thawed & - & $105[13-29]$ & $4 / 5$ & $4 / 4$ \\
& + & $103[20-34]$ & $2 / 5$ & $36(35)^{a b}$ \\
Freeze-dried & - & $119[14-30]$ & $4 / 6$ & $3(2.5)^{a}$ \\
& + & $120[12-24]$ & $11^{*}(9.2)^{a b}$ \\
\hline
\end{tabular}

${ }^{a-c}$ Different superscripts within columns denote significant differences at $p<0.05$.

*Including 1 stillborn offspring. 
participate in full-term development after ICSI. In the mouse, the offspring rate after ICSI with freezedried spermatozoa was comparable to that with frozenthawed spermatozoa (Kusakabe et al., 2001; Ward et al., 2003). However, a significantly lower offspring rate after ICSI with freeze-dried rat spermatozoa was noted $(9.2 \%$ vs $35.0 \%$ in frozen-thawed group; Table 2), suggesting that chromosomal damage and deterioration of the sperm-borne oocyte-activating factor (SOAF) may be induced during the freezedrying process of rat spermatozoa. Since more than $90 \%$ of freeze-dried rabbit spermatozoa carried a normal chromosome set, the impaired development of the rabbit embryos produced by ICSI with freeze-dried spermatozoa has been suggested to result from the deterioration of the SOAF molecules (Liu et al., 2004). If so, assisting oocyte activation prior to ICSI may contribute to improving the offspring rate. Since the tube containing the freeze-dried sperm sample was sealed under air in the present study, the drying sperm may absorb moisture from the air and be oxidized. If cells are exposed to radical oxygen species, some DNA damage may be induced (Potts, 1994). The chromosomal integrity and SOAF activity of freezedried rat spermatozoa, the air-conditioning optimal for freeze-drying, as well as the possibility of long-term storage without a liquid nitrogen tank, remain to be investigated further.

In the present study, the ultrasonic treatment of rat spermatozoa contributed to improving the offspring rate after ICSI (Table 2), suggesting that the sperm membrane disruption induced by sonication may be favorable for the subsequent decondensation of sperm heads and the transformation into male pronucleus, because the ICSI procedure bypasses a series of physiological changes in the membranes. In addition, the oocyte activation rates shown in Table 1 suggest that accelerated interaction of SOAF molecules with ooplasm is associated with the ultrasonic treatment of the spermatozoa. During normal fertilization, the membrane-vesicle-associated membrane proteins (VAMP) are lost from the acrosome-reacted spermatozoa, and the nuclear matrix proteins (NuMA) appear in the decondensing sperm heads (Hewitson et al., 1999). In the rhesus monkey, microinjection of acrosome-intact sperm heads resulted in a delay of sperm head decondensation (Sutovsky et al., 1996). These authors suggested that persistence of VAMP in the sperm heads enclosed by perinuclear theca is responsible for this delay in nuclear decondensation. The PNA lectin-staining employed here (Fig. 1) was not fully effective for interpreting whether the complete removal of membrane-associated components plays an important role in improving the efficiency of ICSI, and further ultrastructural or cytochemical evidence is required.
An interspecies microinsemination assay showed that no rat or bovine spermatozoa decondensed in hamster oocytes even $1 \mathrm{~h}$ after ICSI, while the sperm heads from mouse, chinchilla, hamster and human enlarged within $1 \mathrm{~h}$ (Perreault et al., 1988). In our preliminary study, decondensed rat sperm heads were not observed until at least $2 \mathrm{~h}$ after ICSI (data not shown), suggesting stable nuclear packaging in rat sperm heads. Perreault et al. (1988) reported that the treatment of rat spermatozoa with a disulfide-reducing agent, dithiothreitol (DTT), was partially effective in accelerating decondensation of the sperm heads. In cattle, the in vitro development of ICSI oocytes was improved by pretreatment of the spermatozoa with DTT (Rho et al., 1998). Also in porcine ICSI, failed or abnormal decondensation of sperm chromatin occurs frequently. Treatment of boar spermatozoa with progesterone prior to ICSI was effective in inducing the acrosome reaction and promotion of male pronucleus formation (Katayama et al., 2002). On the other hand, ultrasonic treatment of mouse spermatozoa had a negative impact (Kuretake et al., 1996) or a negligible influence (Tateno et al., 2000) on the developmental competence of ICSI oocytes. The difference in the effect of sonication between the rat and mouse may be due to the different profiles of $\mathrm{p} 34^{\mathrm{cdc} 2}$ kinase kinetics in the ovulated oocytes. The basal level of p34 ${ }^{\text {cdc2 }}$ kinase activity in the rat oocytes was less than half that of the mouse oocytes and further decreased during the process of spontaneous activation, a unique characteristic in rat oocytes (Ito et al., 2005). The ultrasonic treatment of rat sperm heads may destabilize the nuclear packaging as well as the membrane structures, and thus facilitate decondensation even in oocytes with a low p34 $4^{\text {cdc2 }}$ kinase activity. In contrast, Kishigami et al. (2004) reported that pre-activated mouse oocytes with low p34 $4^{\text {cdc2 }}$ kinase activity supported male pronucleus formation after ICSI. This means that mouse sperm head can decondense in conditions of low MPF activity of oocytes, suggesting a difference between mouse and rat.

It is concluded that viable rat offspring can be obtained by intracytoplasmic injection of freeze-dried and rehydrated spermatozoa, and that the ultrasonic treatment of spermatozoa, regardless of the subsequent freeze-thawing or freeze-drying, is effective in increasing the production of ICSI-derived offspring.

\section{Acknowledgements}

This study was supported in part by Grant-in-Aids for the Priority Areas (to M.H., No. 15082211) and the 21st Century COE Program (to S.H.) from the Ministry of 
Education, Culture, Sports, Science and Technology of Japan, and by a Grant-in-Aid for Scientific Research (to M.H., No. 16300139) and a Research Fellowship for Young Scientists (to J.I., No. 8254) from the Japan Society for the Promotion of Science.

\section{References}

Fazeli, A., Hage, W.J., Cheng, F.P., Voorhout, W.F., Marks, A., Bevers, M.M. \& Colenbrander, B. (1997). Acrosome-intact boar spermatozoa initiate binding to the homologous zona pellucida in vitro. Biol. Reprod. 56, 430-8.

Hewitson, L., Dominko, T., Takahashi, D., Martinovich, C., Ramalho-Santos, J., Sutovsky, P., Fanton, J., Jacob, D., Monteith, D., Neuringer, M., Battaglia, D., Simerly, C. \& Schatten, G. (1999). Unique checkpoints during the first cell cycle of fertilization after intracytoplasmic sperm injection in rhesus monkeys. Nat. Med. 5, 431-3.

Hirabayashi, M., Ito, K., Sekimoto, A., Hochi, S. \& Ueda, M. (2001). Production of transgenic rats using young SpragueDawley females treated with PMSG and hCG. Exp. Anim. 50, 365-9.

Hirabayashi, M., Kato, M., Aoto, T., Sekimoto, A., Ueda, M., Miyoshi, I., Kasai, N. \& Hochi, S. (2002a). Offspring derived from intracytoplasmic injection of transgenic rat sperm. Transgenic Res. 11, 221-8.

Hirabayashi, M., Kato, M., Aoto, T., Ueda, M. \& Hochi, S. (2002b). Rescue of infertile transgenic rat line by intracytoplasmic injection of cryopreserved round spermatids. Mol. Reprod. Dev. 62, 295-9.

Hirabayashi, M., Kato, M., Ishikawa, A., Kaneko, R., Yagi, T. \& Hochi, S. (2005). Factors affecting ICSI-mediated DNA transfer: effects of sonication and freeze-thawing, rat strains for sperm and oocyte donors, and different constructs of exogenous DNA. Mol. Reprod. Dev. 70, 422-8.

Ito, J., Hirabayashi, M., Kato, M., Takeuchi, A., Ito, M., Shimada, M. \& Hochi, S. (2005). Contribution of high $\mathrm{p} 34^{\mathrm{cdc} 2}$ kinase activity to premature chromosome condensation of injected somatic cell nuclei in rat oocytes. Reproduction 129, 171-80.

Kaneko, T., Whittingham, D.G., Overstreet, J.W. \& Yanagimachi, R. (2003a). Tolerance of the mouse sperm nuclei to freeze-drying depends on their disulfide status. Biol. Reprod. 69, 1859-62.

Kaneko, T., Whittingham, D.G. \& Yanagimachi, R. (2003b). Effect of $\mathrm{pH}$ value of freeze-drying solution on the chromosome integrity and developmental ability of mouse spermatozoa. Biol. Reprod. 68, 136-9.

Katayama, M., Miyano, T., Miyake, M. \& Kato, S. (2002). Progesterone treatment of boar spermatozoa improves male pronuclear formation after intracytoplasmic sperm injection into porcine oocytes. Zygote 10, 95-104.

Kato, M., Ishikawa, A., Kaneko, R., Yagi, T., Hochi, S. \& Hirabayashi, M. (2004a). Production of transgenic rats by ooplasmic injection of spermatogenic cells exposed to exogenous DNA: a preliminary study. Mol. Reprod. Dev. 69, 153-8.

Kato, M., Ishikawa, A., Hochi, S. \& Hirabayashi, M. (2004b). Effect of activation regimens for rat oocytes on full-term development after round spermatid injection. Contemp. Top. Lab. Anim. Sci. 43/2, 13-15.

Keskintepe, L., Pacholczyk, G., Machnicka, A., Norris, K., Curuk, M.A., Khan, I. \& Brackett, B.G. (2002). Bovine blastocyst development from oocytes injected with freezedried spermatozoa. Biol. Reprod. 67, 409-15.

Kishigami, S., Wakayama, S., Nguyen, VT. \& Wakayama, T. (2004). Similar time restriction for intracytoplasmic sperm injection and round spermatid injection into activated oocytes for efficient offspring production. Biol. Reprod. 70, 1863-9.

Kuretake, S., Kimura, Y., Hoshi, K. \& Yanagimachi, R. (1996). Fertilization and development of mouse oocytes injected with isolated sperm heads. Biol. Reprod. 55, 789-95.

Kusakabe, H., Szczygiel, M.A., Whittingham, D.G. \& Yanagimachi, R. (2001). Maintenance of genetic integrity in frozen and freeze-dried mouse spermatozoa. Proc. Natl. Acad. Sci. USA 98, 13501-6.

Kwon, I.K., Park, K.E. \& Niwa, K. (2004). Activation, pronuclear formation, and development in vitro of pig oocytes following intracytoplasmic injection of freezedried spermatozoa. Biol. Reprod. 71, 1430-6.

Liu, J.L., Kusakabe, H., Chang, C.C., Suzuki, H., Schmidt, D.W., Julian, M., Pfeffer, R., Bormann, C.L., Tian, X.C., Yanagimachi, R. \& Yang, X. (2004). Freeze-dried sperm fertilization leads to full-term development in rabbits. Biol. Reprod. 70, 1776-81.

Ogura, A., Ogonuki, N., Inoue, K. \& Mochida, K. (2003). New microinsemination techniques for laboratory animals. Theriogenology 59, 87-94.

Oh, S.H., Miyoshi, K. \& Funahashi, H. (1998). Rat oocytes fertilized in modified rat 1-cell embryo culture medium containing a high sodium chloride concentration and bovine serum albumin maintain developmental ability to the blastocyst stage. Biol. Reprod. 59, 884-9.

Palermo, G.D., Colombero, L.T. \& Rosenwaks, Z. (1997). The human sperm centrosome is responsible for normal syngamy and early embryonic development. Rev. Reprod. 2, 19-27.

Perreault, S.D., Barbee, R.R., Elstein, K.H., Zucker, R.M. \& Keefer, C.L. (1988). Interspecies differences in the stability of mammalian sperm nuclei assessed in vivo by sperm microinjection and in vitro by flow cytometry. Biol. Reprod. 39, 157-67.

Potts, M. (1994). Desiccation tolerance of prokaryotes. Micro. Rev. 58, 755-805.

Rho, G.J., Kawarsky, S., Johnson, W.H., Kochhar, K. \& Betteridge, K.J. (1998). Sperm and oocyte treatments to improve the formation of male and female pronuclei and subsequent development following intracytoplasmic sperm injection into bovine oocytes. Biol. Reprod. 59, 918-24.

Schatten, G., Simerly, C. \& Schatten, H. (1991). Maternal inheritance of centrosomes in mammals? Studies on parthenogenesis and polyspermy in mice. Proc. Natl. Acad. Sci. USA 88, 6785-9.

Sutovsky, P., Hewitson, L., Simerly, C.R., Tengowski, M.W., Navara, C.S., Haavisto, A. \& Schatten, G. (1996). Intracytoplasmic sperm injection for Rhesus monkey fertilization results in unusual chromatin, cytoskeletal, and membrane events, but eventually leads to pronuclear 
development and sperm aster assembly. Hum. Reprod. 11, 1703-12.

Tateno, H., Kimura, Y. \& Yanagimachi, R. (2000). Sonication per se is not as deleterious to sperm chromosomes as previously inferred. Biol. Reprod. 63, 341-6.

Wakayama, T. \& Yanagimachi, R. (1998). Development of normal mice from oocytes injected with freeze-dried spermatozoa. Nat. Biotechnol. 16, 639-41.
Ward, M.A., Kaneko, T., Kusakabe, H., Biggers, J.D., Whittingham, D.G. \& Yanagimachi, R. (2003). Long-term preservation of mouse spermatozoa after freeze-drying and freezing without cryoprotection. Biol. Reprod. 69, 21008.

Yllera-Fernandez, M.M., Crozet, N. \& Ahmed-Ali, M. (1992). Microtubule distribution during fertilization in the rabbit. Mol. Reprod. Dev. 32, 271-6. 